\title{
EFICIÊNCIA DE USO DE FÓSFORO PELOS GENÓTIPOS DE FEIJÃO
}

\author{
Nand Kumar Fageria ${ }^{1}$
}

\begin{abstract}
RESUMO
A deficiência de fósforo é um dos fatores mais importantes que limitam a produção de feijão em solos de cerrado. Nesta situação, o uso de genótipos eficientes pode ser solução para a melhoria da produção em solos deficientes em fósforo, como de cerrado. Foi conduzido um experimento em casa de vegetação, no Centro Nacional de Pesquisa de Arroz e Feijão, com o objetivo de se avaliar a eficiência do uso de P pelos 15 genótipos de feijão (Phaseolus vulgaris L.) em níveis baixo, médio e alto de P, em um Latossolo Vermelho-Escuro. Diferenças significativas foram obtidas entre genótipos com o uso de fósforo. Os genótipos Rio Doce, São José, IPA 9 e Roxo 9 foram classificados como eficientes e responsivos.
\end{abstract}

Palavras-chave: Phaseolus vulgaris L., produção de matéria seca da parte aérea, comprimento das raízes, nutrição mineral

\section{PHOSPHORUS USE EFFICIENCY BY BEAN GENOTYPES}

\begin{abstract}
Phosphorus deficiency is one of the most yield limiting factors for bean production in savanna soils. Under this situation growing phosphorus efficient genotypes would be a solution to improve crop yields on $\mathrm{P}$ deficient soils such as savanna. A greenhouse experiment was conducted at the National Rice and Bean Research Center to evaluate P use efficiency of 15 bean genotypes (Phaseolus vulgaris L.) to low, medium and high P levels on Dark Red Latosol. Significant differences were found among the genotypes for P use efficiency. Genotypes Rio Doce, São José, IPA 9 and Roxo 9 were classified as efficient and responsive.
\end{abstract}

Key words: Phaseolus vulgaris L., dry matter yield of tops, root length, mineral nutrition

\section{INTRODUÇÃO}

O feijoeiro está concentrado nos trópicos e nos subtrópicos da América Latina (46,74\%), na África, região ao sul do Saara $(24,1 \%)$, na América do Norte $(11,6 \%)$ na Europa $(10,4 \%)$ e na Ásia e África do Norte (6,57\%) (Singh, 1992). Para o Brasil, além de seu caráter econômico, o feijão é um produto de alto significado social, plantado em 5,5 milhões de hectares, com uma produção anual em torno de 3,3 milhões de toneladas.

A produtividade média nacional é de aproximadamente 609 $\mathrm{kg} \cdot \mathrm{ha}^{-1}$, considerada baixa, e a baixa fertilidade do solo de cerrado, especialmente de fósforo, é bastante conhecida. Esses solos possuem teores extremamente pequenos de fósforo e possuem alta capacidade de fixação deste nutriente. Nesta situação, o uso de genótipos eficientes na absorção e na utilização de fósforo pode ser uma solução complementar para aumentar a produtividade e reduzir o custo de produção. É relatado, em literatura, que existe uma diferença significativa entre genótipos de feijão na absorção e utilização de fósforo (Singh et al., 1989; Yan et al., 1995a,b).

O objetivo deste trabalho foi avaliar os genótipos de feijão no uso de fósforo em um Latossolo Vermelho-Escuro de cerrado.

\footnotetext{
${ }^{1}$ Pesquisador, Ph.D., Embrapa Arroz e Feijão, CP 179, 74001-970 Goiânia, GO, fone: (062)833-2178. E-mail: fageria@ cnpaf.embrapa.br
} 


\section{MATERIAL E MÉTODOS}

Foi conduzido um experimento em casa de vegetação, no Centro Nacional de Pesquisa de Arroz e Feijão (CNPAF) em Santo Antônio de Goiás, GO, em maio de 1994, em que o solo utilizado foi um Latossolo Vermelho-Escuro, cuja análise química revelou $\mathrm{pH} 4,9, \mathrm{P}$ 0,8mg. $\mathrm{kg}^{-1}, \mathrm{~K} 36 \mathrm{mg} \cdot \mathrm{kg}^{-1}$, Ca 0,2

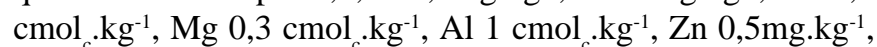
$\mathrm{Cu} 1,3 \mathrm{mg} \cdot \mathrm{kg}^{-1}$, Fe 83mg.kg-1, Mn 16mg.kg-1 e M.O. 21 g.kg-1. A análise granulométrica revelou $470 \mathrm{~g} . \mathrm{kg}^{-1}$ de argila; $260 \mathrm{~g} \cdot \mathrm{kg}^{-1}$ de silte e $270 \mathrm{~g} . \mathrm{kg}^{1}$ de areia e a análise do solo foi realizada segundo rotina adotada pela Embrapa (1997).

Para avaliar a resposta de genótipos de feijão, citam-se três níveis de fósforo no solo; o nível baixo, que corresponde ao teor natural de $\mathrm{P}$ do solo; o nível médio, que recebeu $50 \mathrm{mg}$ P. $\mathrm{kg}^{-1}$ e o nível alto, com 200mg P. $\mathrm{kg}^{-1}$ do solo, através do super fosfato triplo. $\mathrm{O}$ experimento foi conduzido em vasos plásticos com $5 \mathrm{~kg}$ de solo, em que cada vaso recebeu $520 \mathrm{mg}$ de $\mathrm{N}$ na forma de sulfato de amônio, 593mg de $\mathrm{K}$ na forma de cloreto de potássio e $1 \mathrm{~g}$ FTE-BR-12 como fonte de micronutrientes na época de plantio (Fageria et al., 1995) e, também, 6g de calcário; depois, foram incubados por três semanas antes do plantio. $\mathrm{O}$ calcário utilizado possui 37,5\% de $\mathrm{CaO}, 11,6 \%$ de $\mathrm{MgO}, 24 \%$ de $\mathrm{MgCO}_{3}, 67 \%$ de $\mathrm{CaCO}_{3}$ e PRNT, 82\%. O delineamento experimental foi inteiramente casualizado, em fatorial de três níveis de $\mathrm{P}$ x 15 genótipos e os tratamentos foram repetidos três vezes. Após a germinação, foram mantidas quatro plantas por vaso e coletadas 22 dias após o plantio. Após coleta da parte aérea, as raízes foram retiradas com a lavagem de água em cada vaso, lavadas várias vezes com água destilada e medido o comprimento máximo. Para se determinar o peso da matéria seca das raízes e parte aérea, o material foi seco em estufa de $70^{\circ} \mathrm{C}$ e moído e digerido com mistura de ácidos nítrico e perclórico $2: 1$, enquanto o fósforo foi determinado colorimetricamente, pelo método descrito por Moraes \& Rabelo (1986).

\section{RESULTADOS E DISCUSSÃO}

Todos os parâmetros de crescimento e absorção de fósforo foram afetados significativamente $(\mathrm{P}<0.01)$ pelo tratamento com fósforo (Tabela 1); entretanto, genótipos e interações entre fósforo x genótipos não foram significativos, com exceção de peso seco das raízes para o genótipo, significando que a resposta relativa de genótipos aos níveis de $\mathrm{P}$ foi igual em relação aos parâmetros de crescimento e absorção de $\mathrm{P}$, resultados esses também sugerem que qualquer nível de $\mathrm{P}$ pode ser usado para avaliar o genótipo de feijão para uso de fósforo. O P x genótipo

Tabela 1. Significado de valores de F de parâmetros de crescimento e teor e uso de $\mathrm{P}$, de 15 genótipos de feijão

\begin{tabular}{lccc}
\hline Parâmetro da planta & Níveis de P & Genótipos & P x G \\
\hline Peso seco da parte aérea & $* *$ & $\mathrm{~ns}$ & $\mathrm{~ns}$ \\
Peso seco das raízes & $* *$ & $*$ & $\mathrm{~ns}$ \\
Comprimento da raiz & $* *$ & $\mathrm{~ns}$ & $\mathrm{~ns}$ \\
Teor de P na parte aérea & $* *$ & $\mathrm{~ns}$ & $\mathrm{~ns}$ \\
Teor de P na raíz & $* *$ & $\mathrm{~ns}$ & $\mathrm{~ns}$ \\
Eficiência de uso de P & $* *$ & $\mathrm{~ns}$ & $\mathrm{~ns}$ \\
\hline
\end{tabular}

${ }^{*}, * *, \mathrm{~ns}=$ significativo a $5 \%$ e $1 \%$ de probabilidade e não significativo, respectivamente interativo não significativo, mostra que existe estabilidade de adaptação de genótipos de feijão, tanto para o baixo como para 0 alto nível de $P$.

Peso seco da parte aérea, teor de $\mathrm{P}$ na parte aérea e raízes, aumentaram significativamente com o aumento de níveis de fósforo no solo (Tabela 2). O aumento no peso seco da parte

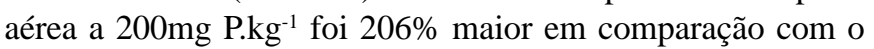
nível zero de P. Desta maneira, o aumento no teor de $\mathrm{P}$ em nível de $200 \mathrm{mg} \mathrm{P} \mathrm{kg}^{-1}$ na parte aérea e nas raízes, foi de $411 \%$ e $150 \%$, respectivamente, em comparação com zero nível de $\mathrm{P}$. O aumento no peso seco das raízes ocorreu em nível de até $50 \mathrm{mg}$ P. $\mathrm{kg}^{-1}$ e houve uma diminuição no peso seco das raízes do alto nível de P. A eficiência de utilização de $\mathrm{P}$ foi menor a $200 \mathrm{mg}$ P. $\mathrm{kg}^{-1}$, em comparação com 50mg P. $\mathrm{kg}^{-1}$ de solo. Os valores de uso de $\mathrm{P}$ geralmente diminuem com o aumento de níveis de nutriente, devido ao nivelamento de produção a alto nível e aumentam em teor e no mesmo nível (Fageria, 1992); isto significa que a eficiência de utilização de $\mathrm{P}$ é máxima a mais baixo nível de nutriente e mínima a mais alto nível.

Tabela 2. Parâmetros de crescimento e teor e uso de P pelos 15 genótipos de feijão, sob três níveis de fósforo ${ }^{1}$

\begin{tabular}{lccc}
\hline \multirow{2}{*}{ Parâmetro da planta } & \multicolumn{3}{c}{ Níveis de P mg.kg-1 } \\
\cline { 2 - 4 } & $\begin{array}{c}\text { Natural do } \\
\text { solo (baixo) }\end{array}$ & $\begin{array}{c}50 \\
\text { (médio) }\end{array}$ & $\begin{array}{c}200 \\
\text { (alto) }\end{array}$ \\
\hline Peso seco da parte aérea (g.vaso) & $1,27 \mathrm{c}$ & $2,74 \mathrm{~b}$ & $3,88 \mathrm{a}$ \\
Peso seco da raiz (g.vaso) & $0,78 \mathrm{c}$ & $1,11 \mathrm{a}$ & $0,91 \mathrm{~b}$ \\
Comprimento da raiz (cm) & $49,00 \mathrm{a}$ & $44,00 \mathrm{~b}$ & $42,00 \mathrm{~b}$ \\
Teor de P na parte aérea (g.kg ${ }^{-1}$ ) & $0,90 \mathrm{c}$ & $4,30 \mathrm{~b}$ & $4,60 \mathrm{a}$ \\
Teor de P na raiz (g.kg $\mathrm{kg}^{-1}$ ) & $1,00 \mathrm{c}$ & $2,00 \mathrm{~b}$ & $2,50 \mathrm{a}$ \\
Eficiência de uso de P (mg peso & & & \\
seco/mg P absorvido) & - & $1384 \mathrm{a}$ & $150 \mathrm{~b}$ \\
\hline
\end{tabular}

${ }^{1}$ Os valores são médias de 15 genótipos. Valores seguidos da mesma letra sob três níveis de fósforo não diferem significativamente a $5 \%$ de probabilidade, pelo teste de Duncan

Com base nos resultados da Tabela 2, pode-se afirmar que, entre os parâmetros de crescimento e absorção de $\mathrm{P}$, que foram analisados, o peso seco da parte aérea e seu teor de P são mais sensíveis à deficiência de $\mathrm{P}$ em um Latossolo Vermelho-Escuro, utilizado no ensaio; esse aumento de peso seco das raízes, que ocorreu em nível de $50 \mathrm{mg}$ P. $\mathrm{kg}^{-1}$, foi de $42 \%$ em comparação com o nível zero de P; por outro lado, o comprimento máximo das raízes ocorreu no nível mínimo de $\mathrm{P}$ e diminuiu com o aumento de nível de $\mathrm{P}$ no solo, significando que em solos deficientes em $\mathrm{P}$, as raízes crescem mais para explorar o maior volume de solo e para satisfazer a necessidade nutricional da planta. Quando a necessidade da planta em nutriente é satisfeita, como no caso de níveis de 50 e $200 \mathrm{mg} \mathrm{P} \mathrm{kg}^{-1}$, as raízes não penetram mais profundamente no solo, fato ligado à demanda $\mathrm{e}$ ao fornecimento de nutriente, além de ser freqüientemente observado em solo de cerrado, com as culturas de feijão, milho, soja e trigo (Fageria et al., 1995).

$\mathrm{O}$ peso da matéria seca da parte aérea de genótipos variou de 2,13 a 3,03g/vaso, o peso seco das raízes variou de 0,62 a $1,16 \mathrm{~g}$.vaso ${ }^{-1}$, o comprimento máximo foi de 42 a $48 \mathrm{~cm}$, o teor de P na parte aérea vacilou de 3,1 a $3,5 \mathrm{~g} \cdot \mathrm{kg}^{-1}$, e o teor de P nas raízes variou de 1,7 a $2,1 \mathrm{~g} . \mathrm{kg}^{-1}$ enquanto a eficiência de utilização de $\mathrm{P}$ ficou de 500 a $1306 \mathrm{mg}$ peso seco por mg $\mathrm{P}$ absorvido (Tabela 3). Entre esses parâmetros, maior variação aconteceu na utilização de $\mathrm{P}$, pelos genótipos de feijão, seguido pelo peso seco da parte aérea e pelo teor de P na parte aérea. 
Tabela 3. Parâmetros de crescimento, teor e uso de fósforo por 15 genótipos de feijão ${ }^{1}$

\begin{tabular}{|c|c|c|c|c|c|c|}
\hline Genótipos & $\begin{array}{c}\text { Peso seco da } \\
\text { parte aérea } \\
\left(\text { g.vaso }{ }^{-1}\right)\end{array}$ & $\begin{array}{l}\text { Peso seco } \\
\text { das raízes } \\
\left(\text { g.vaso }^{-1}\right)\end{array}$ & $\begin{array}{l}\text { Comprimento } \\
\text { da raiz } \\
(\mathrm{cm})\end{array}$ & $\begin{array}{c}\text { Teor de P na } \\
\text { parte aérea } \\
\left(\mathrm{g} \cdot \mathrm{kg}^{-1}\right)\end{array}$ & $\begin{array}{l}\text { Teor de } \mathrm{P} \\
\text { na raiz } \\
\left(\mathrm{g} \cdot \mathrm{kg}^{-1}\right)\end{array}$ & $\begin{array}{c}\text { Eficiência de uso de } \\
\text { P (mg peso seco/mg } \\
\text { P acumulado) }\end{array}$ \\
\hline 1.Aporé & $2,93 \mathrm{ab}$ & $0,89 \mathrm{abc}$ & $45 \mathrm{ab}$ & $3,3 \mathrm{ab}$ & $1,9 a b c$ & $615 b$ \\
\hline 2.Goitacazes & $2,58 \mathrm{abc}$ & $0,98 \mathrm{ab}$ & $48 \mathrm{a}$ & $3,1 \mathrm{~b}$ & $1,8 \mathrm{bc}$ & $709 b$ \\
\hline 3.Carioca-MG & $2,64 a b c$ & $0,91 \mathrm{abc}$ & $43 \mathrm{ab}$ & $3,3 \mathrm{ab}$ & $1,8 \mathrm{bc}$ & $739 b$ \\
\hline 4.Carioca-IAC & $3,03 a$ & $1,16 \mathrm{a}$ & $46 a b$ & $3,5 \mathrm{a}$ & $1,7 \mathrm{c}$ & $687 b$ \\
\hline 5.Rio Doce & $2,61 \mathrm{abc}$ & $0,96 a b$ & $46 a b$ & $3,4 \mathrm{ab}$ & $1,8 \mathrm{bc}$ & $906 a b$ \\
\hline 6.Emgopa 201-Ouro & $2,37 \mathrm{bc}$ & $0,82 b c$ & $42 b$ & $3,3 \mathrm{ab}$ & $2,2 \mathrm{a}$ & $522 b$ \\
\hline 7.IPA 6 & $2,67 a b c$ & $1,07 \mathrm{ab}$ & $47 \mathrm{ab}$ & $3,3 \mathrm{ab}$ & $1,8 \mathrm{bc}$ & $680 \mathrm{~b}$ \\
\hline 8.São José & $2,66 a b c$ & $0,94 a b$ & $46 a b$ & $3,1 b$ & $1,7 \mathrm{c}$ & $937 \mathrm{ab}$ \\
\hline 9.IPA 9 & $2,50 \mathrm{abc}$ & $1,01 \mathrm{ab}$ & $42 b$ & $3,3 \mathrm{ab}$ & $1,7 \mathrm{c}$ & $1306 a$ \\
\hline 10.Onix & $2,58 \mathrm{abc}$ & $0,77 \mathrm{bc}$ & $46 \mathrm{ab}$ & $3,3 \mathrm{ab}$ & $1,8 \mathrm{bc}$ & $968 \mathrm{ab}$ \\
\hline 11.Xodó & $2,13 \mathrm{c}$ & $0,62 \mathrm{c}$ & $43 a b$ & $3,1 b$ & $1,8 \mathrm{bc}$ & $760 \mathrm{~b}$ \\
\hline 12.Serrano & $2,51 \mathrm{abc}$ & $1,00 \mathrm{ab}$ & $42 \mathrm{ab}$ & $3,1 \mathrm{~b}$ & $1,7 \mathrm{c}$ & $623 b$ \\
\hline 13.Pot 51 & $2,89 \mathrm{ab}$ & $0,87 \mathrm{abc}$ & $45 \mathrm{ab}$ & 3,3ab & $1,9 \mathrm{abc}$ & $1023 a b$ \\
\hline 14.Safira & $2,67 a b c$ & $0,99 \mathrm{ab}$ & $46 a b$ & $3,2 b$ & $1,8 b c$ & $500 \mathrm{~b}$ \\
\hline 15.Roxo 9 & 2,71abc & $1,00 \mathrm{ab}$ & $47 \mathrm{ab}$ & $3,2 b$ & $2,1 \mathrm{ab}$ & $528 \mathrm{~b}$ \\
\hline
\end{tabular}

${ }^{1}$ Os valores são médias de três níveis de fósforo. Valores seguidos da mesma letra na mesma coluna não diferem significativamente a $5 \%$ de probabilidade, pelo teste de Duncan

$$
{ }^{2} \text { Eficiência de uso de P }=\frac{\begin{array}{c}
\text { Produção de matéria seca } \\
\text { da parte aérea e raízes com } \\
\text { médio e alto nível de P }
\end{array}}{\begin{array}{c}
\text { Acumulação total (parte } \\
\text { aérea + raízes) de P com } \\
\text { médio e alto nível de P }
\end{array}}-\frac{\begin{array}{c}
\text { Produção de matéria seca } \\
\text { da parte aérea e raízes com } \\
\text { baixo nível de } \mathrm{p}
\end{array}}{\begin{array}{c}
\text { Acumulação total (Parte } \\
\text { aérea + raízes) de P com } \\
\text { baixo nível de P }
\end{array}}
$$

A eficiência de uso de fósforo, definido como sendo o produto da matéria seca (parte aérea mais raízes) em mg pela quantidade de $\mathrm{P}$ absorvido (acumulação de $\mathrm{P}$ na parte aérea e raízes) variou significativamente entre genótipos (Tabela 3). A grande diferença entre genótipos de feijão na absorção e utilização de $\mathrm{P}$ já havia sido constatada (Yan et al., 1995a,b). Os resultados de utilização de $\mathrm{P}$ foram relacionados com a produção de matéria seca (parte aérea mais raízes) a baixo nível de $\mathrm{P}\left(0 \mathrm{mg} \mathrm{P} \mathrm{kg}^{-1}\right)$ e ao uso de $\mathrm{P}$ a médio nível de $\mathrm{P}$ (50mg P.kg-1) como sugerido por Fageria \& Baligar (1993) para classificação de genótipos eficientes e responsivos, ineficientes e não responsivos (Figura 1).

A produção média de 15 genótipos a baixo nível de $\mathrm{P}$ e a média de eficiência de 15 genótipos na utilização de $\mathrm{P}$, foram usadas como índices de separação de genótipos, em quatro grupos:

1) genótipos eficientes e responsivos (GER): representam os genótipos que produziram acima da média de 15 genótipos sob condições de baixo nível de fósforo e responderam bem ao incremento na produção, a alto nível do nutriente. Genótipos como Rio Doce, São José e IPA 9 são classificados neste grupo;

2) genótipos eficientes e não responsivos (GENR): representam os genótipos que produziram acima da média de 15 genótipos em baixo nível de fósforo, mas não responderam ao nível alto desse nutriente. Os genótipos classificados neste

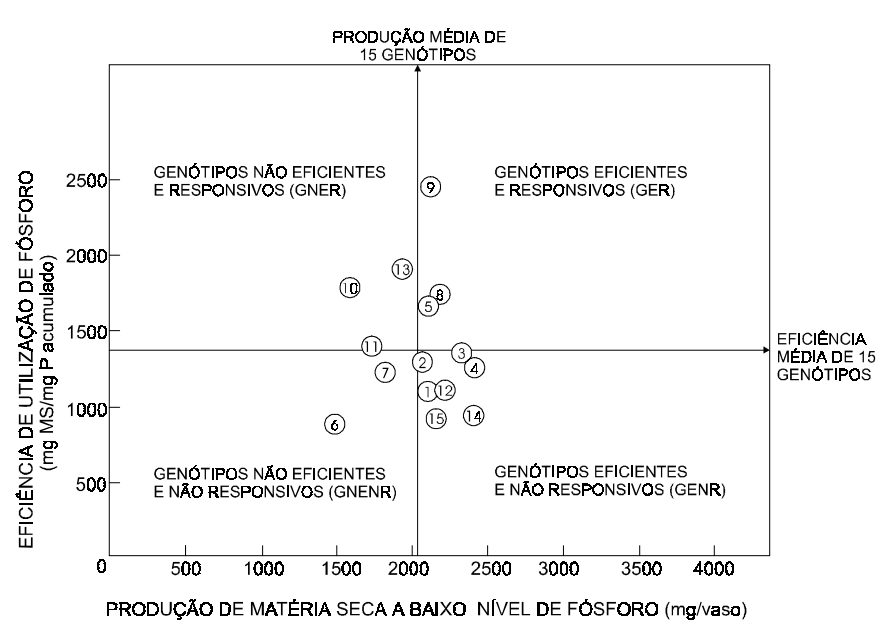

Figura 1.Classificação de genótipos de feijão no uso de fósforo; os números correspondem aos genótipos numerados na Tabela 3

grupo, são: Aporé, Goytacazes, Carioca-MG, Carioca IAC, Serrano, Roxo 9 e Safira.

3) genótipos não eficientes e responsivos (GNER): representam os genótipos que produziram abaixo da média, sob baixo nível de fósforo, mas apresentam apreciável incremento na produção a alto nível de fósforo. Os genótipos Onix, Xodo e Pot 51 são classificam-se neste grupo;

4) genótipos não eficientes e não responsivos (GNENR): representam os genótipos que produziram abaixo da média, em baixo ou em alto nível de fósforo. Os genótipos EMGOPA 201-Ouro e IPA 6 foram classificados neste grupo.

Do ponto de vista prático, os genótipos que produziram bem a baixo nível de $\mathrm{P}$ e responderam bem com a aplicação de $\mathrm{P}$, são considerados melhores. 


\section{CONCLUSÕES}

1. Os genótipos de feijão apresentaram comportamento diferenciado com relação à eficiência de utilização de fósforo.

2. Os genótipos Rio Doce, São José e IPA 9 foram considerados eficientes e responsivos, e Aporé, Goytacazes, Carioca-MG, Carioca-IAC, Serrano, Safira e Roxo 9, eficientes e não responsivos, na utilização de fósforo.

3. Os genótipos Onix, Xodo e Pot 51 foram classificados como ineficientes e responsivas, e EMGOPA-201-Ouro e IPA 6 , ineficientes e não responsivos.

4. A produção de matéria seca da parte aérea e o teor de P na parte aérea foram mais sensíveis à deficiência de fósforo, o que permitiu concluir que foram mais apropriadas para avaliar os genótipos de feijão no uso de fósforo em casa de vegetação.

\section{REFERÊNCIAS BIBLIOGRÁFICAS}

EMBRAPA. Serviço Nacional de Levantamento e Conservação de Solos (Rio de Janeiro, RJ). Manual de métodos de análise de solo. 2.ed. Rio de Janeiro, 1997. 212p.

FAGERIA, N.K. Maximining crop yields. New York: Marcel Dekker, 1992. 274p.
FAGERIA, N.K; BALIGAR, V.C. Screening crop genotypes for mineral stresses. In: WORKSHOP ON ADAPTATION OF PLANTS TO SOIL STRESSES, 1993, Lincoln. Proceedings. Lincoln: INSTOR MIL, 1993. p.142-159.

FAGERIA, N.K; ZIMMERMANN, F.J.P; BALIGAR, V.C. Lime and phosphorus interaction on growth and nutrient uptake by upland rice, wheat, common bean and corn in on oxisol. Journal of Plant Nutrition, New York, v.18, p.25192532, 1995.

MORAES, J.F.V; RABELO, N.A. Um método simples para a digestão de amostra de plantas. Goiânia: EMBRAPACNPAF, 1986. 12p. (EMBRAPA-CNPAF. Documentos, 12).

SINGH, S.P. Common bean improvement in the tropics. Plant Breeding Review, New York, v.10, p.199-269, 1992.

SINGH, S.P.; URREA, C.A.; GUTIERREZ, J.A.; GARCIA, J. Selection for yield at two fertilizer levels in small-seeded common bean. Canadian Journal of Plant Science, Ottawa, v.65, p.1011-1017, 1989.

YAN, X; LYNACH, J.P; BEEBE, S.E. Genetic variation for phosphorus efficiency of common bean in constrasting soil types. I. Vegetative response. Crop Science, Madison, v.35, p.1086-1093, 1995a.

YAN, X; BEEBE, S.E; LYNCH, J.P. Genetic variation for phosphorus efficiency of common bean in constrasting soil type. II. Yield response. Crop Science, Madison, v.35, p.1094-1099, 1995b. 